\title{
Laparoscopic single trocar appendectomy
}

\author{
Mohamed A Sharaan, MD; Samer S Bessa, MD; Ahmed El-Gendy, $M D$; \\ Alaa H Abdel-Razek, MD, FRCS
}

Department of General Surgery, Alexandria University, Alexandria, Egypt.

\author{
Corresponding Author: \\ Mohamed Abdallah Sharaan, Department of General Surgery, Faculty of \\ Medicine, Alexandria University, Egypt.
}

\begin{abstract}
Background and objective: Single port surgery is a new surgical technique with more promising advantages of minimal access laparoscopy. The present study was to assess the feasibility of the single trocar appendectomy, its morbidities and satisfaction for both patients and surgeons.

Methods: Consecutive patients presenting with acute appendicitis were treated by single port appendectomy. Through an umbilical incision, the single port was inserted, we used SILS port (Covidien) in 15 cases and Xcone port (Karl Storz) in 27 cases. A $5 \mathrm{~mm} 30^{\circ}$ lens was used. The mesoappendix was coagulated and divided by Ligasure, Harmonic scalpel or diathermy. Absorbable endoloops were used to ligate the base of the appendix. Assessment included operative time, complications, patient satisfaction and surgeon satisfaction.

Results: The study included 42 patients, 24 (57.1\%) females and 18 (42.9\%) males with a mean age of $27.3 \pm 5.4$ years. The procedure was completed in all of the patients. Complications occurred in 4 patients (9.5\%). The mean hospital stay was 1.1 days \pm 1 . The procedure achieved accepted cosmetic outcome with minimal or hidden scar. The patients were satisfied as good or very good procedure. The surgeons found it satisfactory in $67.6 \%$ of operations as regards technical difficulties and outcome.

Conclusion: The procedure is feasible and safe. Neither mortality nor visceral injury occurred in this study. Rate of complications was 9.5\%. Accepted rate of satisfaction was present for both patients and surgeons. It achieved accepted cosmetic results with minimal or hidden scar. Complications are expected to decrease by improvement in the learning curve.
\end{abstract}

Key words: single port; appendectomy; laparoscopy.

\section{Introduction:}

Acute appendicitis is a common intraabdominal inflammatory disease which requires emergency surgery as the most frequent therapeutic scenario. Since the introduction of laparoscopic appendectomy, it has become an alternative method of treatment to open appendectomy because of decreased postoperative pain, better cosmetic result, and rapid return to the daily activities. ${ }^{1}$
During the era of laparoscopic surgery, there has been a common trend towards less invasive techniques. A natural extension of this trend is to perform operations without scars. The most prominent techniques are transumbilical single-incision laparoscopic surgery (SILS) and natural orifice transluminal endoscopic surgery (NOTES). ${ }^{2}$ As the latter is still confronted with some technical and instrumental difficulties, 
SILS seems to be more ready for wider use in surgical practice. There are reliable equipments available for SILS procedures. However, the operative technique, although different from conventional laparoscopy, is probably easier to be learned compared to NOTES technique. 1,2 A number of advantages have been proposed related to this approach including better cosmesis (abdominal surgery performed through a hidden umbilical incision) and the ability to convert to standard multiport laparoscopic surgery if needed without denying the disadvantage and complication related to this new technique.3,4 SILS appendectomy may be even more advantageous to the patients by eliminating the scars. However, the role of the SILS appendectomy is still evolving ${ }^{5-7}$ More studies evaluating the technique in different clinical situations as well as randomized controlled trials are needed in order to assess the real benefits of the SILS appendectomy in general surgical practice.

The aim of the present study was to study the feasibility of the SILS appendectomy, its morbidities and satisfaction of both patients and surgeons.

\section{Material and methods:}

This study was approved by the Ethics Committee in Faculty of Medicine, Alexandria University. Between March 2009 and April 2011, 42 patients were subjected to Single Incision Laparoscopic Appendectomy (SILA). These patients presented with acute abdominal pain and they were all diagnosed clinically as acute appendicitis. In suspicious cases the diagnosis was confirmed by abdominal ultrasound (US) and/or computed tomography $(\mathrm{CT})$. Exclusion criteria included patients with complicated appendicitis (perforated appendix, appendicular mass or abscess), patients with previous lower abdominal surgery and pregnant patients. The surgical team who performed all these cases were familiar with the SILS instruments and they had performed SILS on animal models. Aninformed consent was signed by the patients before surgery. All our patients received intra-venous one gram third generation
Cephalosporin and 500mg Metronidazole at the induction of anesthesia. All the patients were placed in the supine position, the right arm was abducted $90^{\circ}$ to the body while the left arm was alongside the body. The camera man and the surgeon were in the left side of the patient while the monitor was placed in the right side of the patient near to the right iliac fossa. Under general anesthesia, an incision was performed about $15 \mathrm{~mm}$ inside of the umbilicus itself and not beyond the umbilical circumference, opening in layers till reaching the linea alba and the peritoneum which were opened by monopolar diathermy. The single port device was then inserted, we used SILS port (Covidien) in 15 cases and Xcone port (Karl Storz) in 27 cases. A 5mm 300 lens was used and laparoscopic exploration was performed. The operating table was tilted to a $30^{\circ}$ Trendelenburg position with a left rotation to allow adequate exposure of the ceacum and the ileo-ceacal junction. We used conventional straight instruments in 27 cases and articulating instruments in 15 cases to facilitate the procedure. The mesoappendix was coagulated and divided near to the appendix using bipolar cautery or LigaSure (Covidien) or Harmonic Scalpel (Ethicon, Johnson \& Johnson) till reaching the junction of the appendix with the ceacum. The base of the appendix was ligated using 2 ligatures of absorbable suture material (Endoloop; Ethicon Endosurgery). Division was then done to the appendix sharply by scissor soaked with betadine. The appendix was then retrieved in a plastic sac through the single port through the umbilical scar without touching the wound.

Follow up was done for early and late postoperative period to assess any complications and postoperative pain. Satisfaction of patients and surgeons was assessed. We assessed the post-operative pain by asking the patients about score of pain from 0 to 10 . Score from Zero to 2 was considered minimal pain, 3 to 5 was considered mild pain, 6 to 8 was considered moderate pain, and 9 to 10 was considered severe pain. Also, patients' satisfaction in this study was assessed by asking the patients to 
give a score from 0 to 10 . Score from Zero to 3 was considered bad, 4 to 6 was considered good, 7 to 9 was considered very good. Surgeon satisfaction was assessed by the surgeons comment on technical difficulties and morbidities.

\section{Results:}

The study included 42 patients, 24 (57.1\%) females and 18 (42.9\%) males with a mean age of $27.3+5.4$ years (range $14-$ 46 years). The mean body mass index was $30.9+7.3 \mathrm{~kg} / \mathrm{m}^{2}$ (ranged from $21-46 \mathrm{~kg} / \mathrm{m}^{2}$. We had 15 obese cases (BMI $>30 \mathrm{~kg} / \mathrm{m}^{2}$ ). Preoperative data are shown in Table(1). All these patients had a preoperative diagnosis of non-complicated acute appendicitis. Ten patients had collection discovered by ultrasound, and 32 patients had low grade fever preoperatively. Two patients had paraumbilical hernias which were operated on

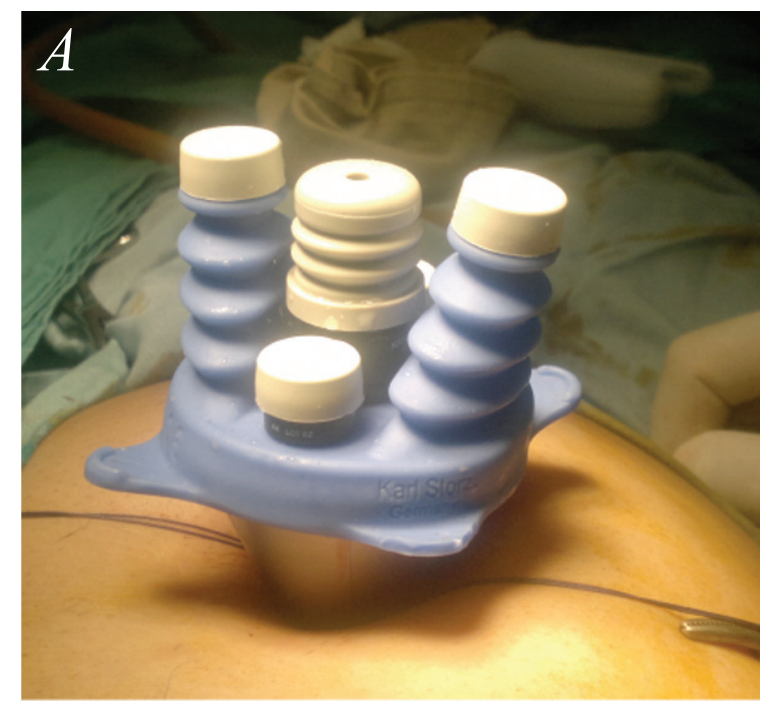

Figure (1): Single port used (A. X cone of Karl Storz) (B. SILS port of Covidien).

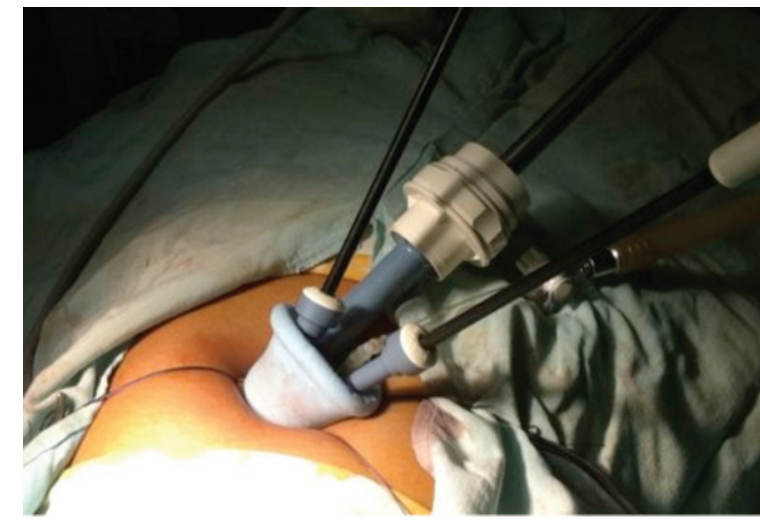

Figure (3): Instruments application to single port. with the SILS procedure. The mean operative time was $48.2+24.5 \mathrm{~min}$ (range, $30-100 \mathrm{~min}$ ). No operative complications (hemorrhage, bowel perforation) were reported. The operative difficulties were recorded in 7 cases, in 5 patients, the difficulty was due to leakage of gas from the single port, while the other 2 difficulties were due to the search of the appendix which was hidden due to inflammatory adhesions. Postoperatively, oral liquids were started within 6 hours and a soft diet within $12 \mathrm{~h}$. Only one dose of parenteral analgesia was administered followed by oral analgesics. Complications occurred in 4 patients (9.5\%). Table(2). Post-operative wound infection occurred in $4.7 \%$ ( 2 out of 42 patients) they were treated by frequent dressing and antibiotics. There was no mortality. The postoperative trocar site hernia was detected in 1 out of 42 cases $(2.3 \%)$. This was operated on by hernia repair
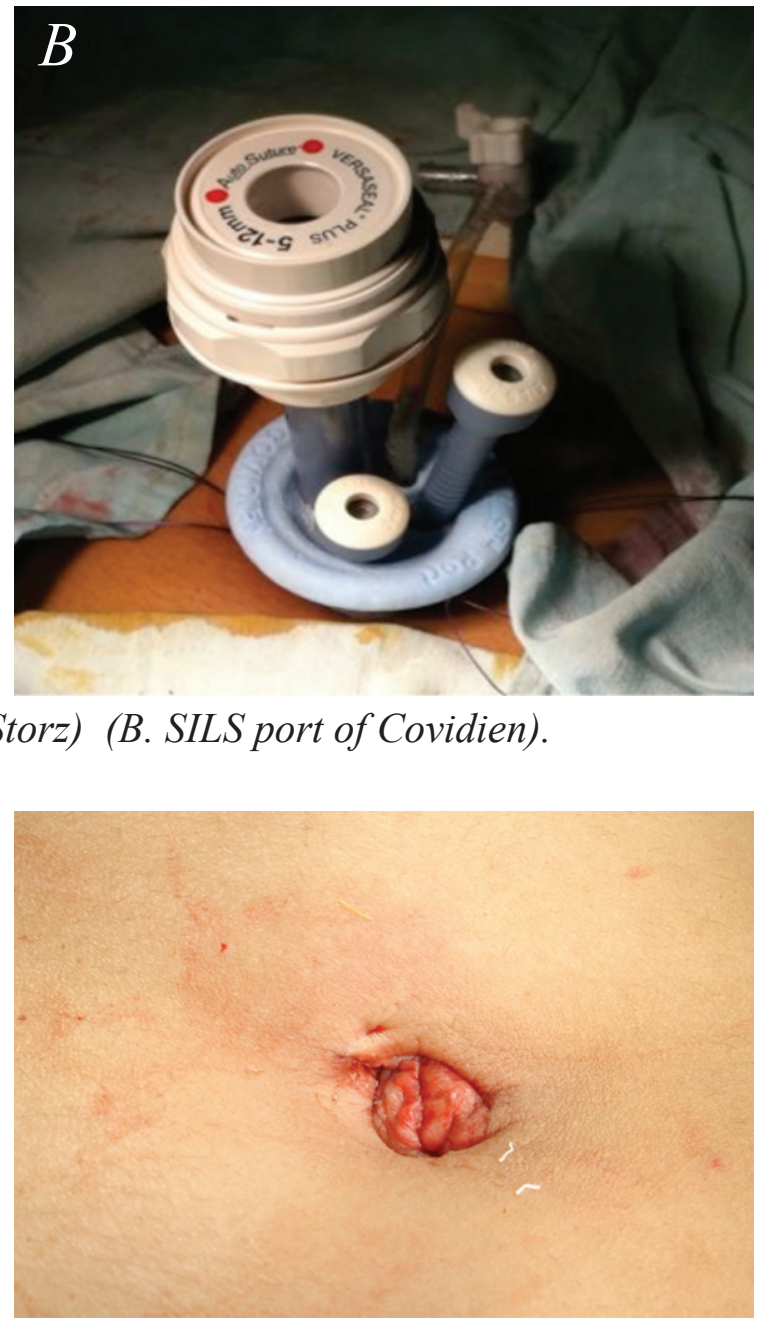

Figure (4): Umbilical scar left at the end of the operation. 
Table (1): Preoperative data of the patients.

\begin{tabular}{|r|c|c|}
\hline & Number of patients $(\mathbf{n}=\mathbf{4 2})$ & $\mathbf{\%}$ \\
\hline Gender & 24 & 57.1 \\
Fale & 18 & 42.9 \\
\hline Body mass index & & \\
$\leq 30 \mathrm{Kg} / \mathrm{m}^{2}$ & 27 & $64.2 \%$ \\
$>30 \mathrm{Kg} / \mathrm{m}^{2}$ & 15 & $35.8 \%$ \\
\hline Associated co-morbidities & & \\
Present & 4 & 9.5 \\
Absent & 38 & 90.5 \\
\hline
\end{tabular}

Table (2): Operative and postoperative data.

\begin{tabular}{|c|c|c|}
\hline & Number of patients $(\mathrm{n}=42)$ & $\%$ \\
\hline Postoperative complications & (Total 5 patients) & (Total: 11.9\%) \\
Ileus & 3 & 7.1 \\
Wound infection & 2 & 4.6 \\
Port site hernia & 1 & 2.3 \\
Minimal & 18 & 42.8 \\
Mild & 24 & 57.2 \\
Postoperative pain score & 14 & 33.3 \\
Vory good & 20 & 66.6 \\
Bad & 0 & 0 \\
\hline Patient satisfaction score & 11 & 26.2 \\
\hline Not easy & & \\
Sifficulty) & & \\
\hline
\end{tabular}


and prolene mesh application. The mean hospital stay of this study was 1.1 days; only 4 cases $(9.5 \%)$ had hospital stay of 2 days because of post-operative ileus that delayed their discharge. No cases of post-operative fistula or post-operative abscess were reported. In this study, patients' satisfaction was assessed. The results of postoperative pain score, patients' satisfaction and other data are shown in Table(2). We also assessed satisfaction of the surgeons who performed the operations. We reported that in 11 cases $(26.1 \%)$ this procedure was technically difficult due to narrow operative field and collision of instruments with adherent hidden severely inflamed appendix, and in 31 cases $(73.8 \%)$ this procedure was relatively easier. There was no conversion to open or standard laparoscopy in all of the cases of this study.

\section{Discussion:}

Appendectomy is the most common abdominal operation in the western world. Laparoscopic appendectomies are currently preferred due to the fact that it offers advantages to patients in terms of more accurate diagnosis, diminished wound infections, and more rapid recovery. ${ }^{8}$ SILS appendectomy may result surely in better cosmesis but probable additional benefits, in terms of more rapid recovery and less post-operative pain, these have not been proven scientifically. However, randomized controlled clinical trials are urgently needed to define the role of SILS appendectomy in the modern surgical armamentarium. Always when a new technique is introduced, the focus should be concentrated on the feasibility, safety, and clinical advantage of the method. Moreover, safety of the procedure is highly dependent on how easily the new technique can be learned by average surgeons. It is expected from a new technique that it can be associated with an increased risk of complications emphasizing the importance of thorough training and education by the interested surgeons. The SILS technique differs from the standard laparoscopic technique remarkably by the use of the single port, specially designed grasping and dissecting instruments, also the access and the application of the single port in the umbilicus. These differences make the procedure more challenging and initiating new learning curve for surgeon.

Standard laparoscopic appendectomy is usually done by using 2 instruments and a $10 \mathrm{~mm}$ camera, the same in SILS appendectomy procedure but through a single port, also the use of articulating instruments in SILS procedure can facilitate the technique. Furthermore SILS appendectomy is relatively easy operation that can be performed properly by one straight instrument and one curved instrument.

When performing appendectomy, one must be prepared for different abdominal findings, like thickened appendix, gangrenous, perforated with peritonitis, or even with pelvic abscess. In these situations the technique of appendectomy should be selected. In this study we had easy and difficult cases, all our 42 patients were operated on by SILS technique without conversions or adding ports. All of the patients in this study had an uneventful recovery. Moreover, the mean operating time was $48 \pm 24.5$ minutes (range $30-100 \mathrm{~min}$ ) comparing well to the operating time in other studies (mean 40min, range 35-102minutes $)^{8}$ and that of the conventional laparoscopic appendectomy in our hospital (mean 43 minutes, range 18-103). ${ }^{8}$

From our experience in SILS procedure, we believe that it is feasible for different kinds of appendicitis, with the possibility of conversion to conventional laparoscopic appendectomy. Also it can be useful in case of double pathology, like appendicitis and cholecystitis, appendicitis and ovarian cyst, also in wrong diagnosis for exploratory laparoscopy. In this study we found that access into the peritoneal cavity and insertion of the single port is more difficult in obese patients, compared to non-obese patients. According to literature especially obese patients benefit from laparoscopic appendectomy compared to open one and laparoscopy should be the preferred technique for these patients. ${ }^{8-10}$ It is, thus, important that new mini-invasive operative techniques like SILS are suitable 
for this patient population too.

Wong et al, ${ }^{11}$ reported in a recent prospective case control study, a decrease postoperative pain after single port laparoscopic cholecystectomy as compared to conventional laparoscopic cholecystectomy. Bucher et $\mathrm{al}, 12$ also reported recent randomized clinical trial, resulting in a reduced post-operative pain in SILS cholecystectomy compared to conventional laparoscopic cholecystectomy. In our study the post-operative pain was in $57.2 \%$ mild and in $42.8 \%$ minimal, a prospective randomized trial of SILS versus laparoscopic appendectomy is needed to clarify post-operative pain difference. In SILA we used an umbilical incision of 15$\mathrm{mm}$, leaving virtually no scar. Using a single port for laparoscopic appendectomy in this study was not a real problem except for a minority of patients in whom we used flexible and rotating instruments (i.e., scissors, graspers, dissectors). Moreover, tilting the operating table enabled us to achieve adequate exposure and dissection. Appendix extraction was through the umbilical incision with protection (plastic sac, the single port itself). In our study, the incidence of port-site infection was $4.7 \%$ ( 2 out of 42 patients), not greater than in other series of LA. We reported in this study an incidence port site hernia of about $2.3 \%$, this may be due to the fact that we did a big incision in the umbilical scar to adapt the single port size, and the difficulty of perfect closure of the rectus sheath because of the depth of the incision and obesity. Tonouchi et al ${ }^{13}$ reported in their review article about trocar site hernia, that risk factors for occurrence of trocar site hernias are, the large trocar size, non-closure or bad closure of the fascial defect, the location if umibilical or paraumbilical, the stretching of the port site for retrieval, obesity, poor nutrition and wound infection. Fransen et al ${ }^{14}$ reported that in SILS the risk of hernia is increased because of the widening of the incision, in addition to the application of the single port which exerts more pressure on the tissue, causing more damage and leading to possible more herniation at the entry site. Our trocar site hernia case was our first case, and it might be related to more widening of the umbilical incision and retraction in the beginning of the study.

The SILS appendectomy approach, is considered as "Embryonic NOTES" appendectomy using an embryologic natural orifice (the umbilicus) as a sole site of abdominal access and is a sure and feasible approach for selected patients with acute appendicitis. Its main aim is to improve the post-operative outcomes (shorter hospital stay, faster return to activity, better cosmesis) by reducing the size and number of incisions. ${ }^{15}$

Although SILS technique looks promising and offers some potential benefits for patients compared to conventional laparoscopy, two possible disadvantages should be considered. SILS technique may be associated with increased risk of hernias. The technique necessitates fascial incision through the abdominal midline that has been considered to be prone to hernia formation. Further, the fascial incision is more traumatic compared to 5 or $12 \mathrm{~mm}$ trocar wounds made with dilating trocars. The second possible disadvantage is the presumed additional costs caused by the procedure-specific port and instruments. These extra operative costs should be taken into account in the current trend towards cost-effectiveness in HealthCare. After introduction of re-usable (autoclavable) instruments for SILS port and instruments, the cost problem could be dealt with. Unfortunately, we did not assess cost effect in this study, it should be assessed in a comparison with standard laparoscopic appendectomy in future studies.

\section{Conclusion:}

SILS technique is technically feasible and safe for the appendectomy procedure. It offers accepted rate of satisfaction for both patients and surgeons. The technique may offer evident cosmetic benefit; it is feasible in obese patients but with more difficult access to the peritoneal cavity. SILA have some complications related to SILS technique, like hernia and wound infection. Appendectomy is a suitable procedure for the training of SILS technique; laparoscopic surgeons starting 
SILS should be well motivated and devoted to this new procedure until learning curve will be established. The SILS procedure may have few disadvantages and its true benefit remains to be shown by randomized controlled trials.

\section{References:}

1- Pedersen A, Petersen O, Wara P, et al: Randomized clinical trial of laparoscopic versus open appendicectomy. Br J Surg 2001; 88: 200-205.

2- Giday S, Kantsevoy S, Kallo A: Principle and history of natural orifice translumenal endoscopic surgery (NOTES). Minim Invasive Ther Allied Technol 2006; 15: 373-377.

3- Ersin S, Firat O, Sozbilen M: Singleincision laparoscopic cholecystectomy: Is it more than a challenge? Surgical Endoscopy and Other Interventional Techniques 2010; 24(1): 68-71.

4- Chow A, Purkayastha S, Paraskeva P: Appendicectomy and cholecystectomy using single-incision laparoscopic surgery (SILS): The first UK experience. Surg Innov 2009; 16: 211-217.

5- Vettoretto N, Mandala V. Single port laparoscopic appendectomy: Are we pursuing real advantages. World Journal of Emergency Surgery 2011; 6: 25.

6- Kim H, Lee I, et al. Single port laparoscopic appendectomy: 43 consecutive cases. Surg Endosc 2010; 24(11): 2765-2769.

7- Chiu C, Ngugen N, Bloom S: Single incision laparoscopic appendectomy using conventional instruments: An initial experience using a novel technique. Surg Endosc 2011; 25: 1153-1159.
8- MG Corneille, MB Steigelman, JG Myers, et al: Laparoscopic appendectomy is superior to open appendectomy in obese patients. American Journal of Surgery 2007; 194(6): 877-881.

9- Varela J, Hinojosa M, Nguyen N: Laparoscopy should be the approach of choice for acute appendicitis in the morbidly obese. American Journal of Surgery 2008; 196(2): 218-222.

10-Chow A, Purkayastha S, Paraskeva P: Appendicectomy and cholecystectomy using single-incision laparoscopic surgery (SILS): The first UK experience. Surgical Innovation 2009; 16(3): 211-217.

11-Wong J, Cheung Y, Chong C: Comparison of post-operative pain between singleincision laparoscopic cholecystectomy and conventional laparoscopic cholecystectomy: Prospective case control study. Surg Laparosc Percut tech 2012; 22(1): 25-28.

12-Bucher P, Pugin F, Buchs N: Randomized clinical trial of laparoendoscopic single site versus conventional laparoscopic cholecystectomy. Br J Surg 2011; 12: 1695-1702.

13-Tonouchi H, Ohmori Y, Kobayashi M, et al: Trocar site hernia (Review article). Arch Surg 2004; 139: 1248-1256.

14-Fransen S, Stassen L, Bouvy N: Single incision laparoscopic cholecystectomy: A review on the complications. $J$ Minim Acess Surg 2012; 8(1): 1-5.

15-Chouillard E, Dache A, Torcivia A: Singleincision laparoscopic appendectomy for acute appendicitis: A preliminary experience. Surg Endosc 2010; 24(8): 1861-1865. 